Article

\title{
Total Roman Domination Number of Rooted Product Graphs
}

\author{
Abel Cabrera Martínez ${ }^{1, * \mathbb{D}}$, Suitberto Cabrera García ${ }^{2}$, Andrés Carrión García ${ }^{2} \mathbb{D}$ and \\ Frank A. Hernández Mira ${ }^{3}$ \\ 1 Departament d'Enginyeria Informàtica i Matemàtiques, Universitat Rovira i Virgili, Av. Països Catalans 26, \\ 43007 Tarragona, Spain \\ 2 Departamento de Estadística e Investigación Operativa Aplicadas y Calidad, \\ Universitat Politécnica de Valencia, Camino de Vera s/n, 46022 Valencia, Spain; \\ suicabga@eio.upv.es (S.C.G.); acarrion@eio.upv.es (A.C.G.) \\ 3 Centro de Ciencias de Desarrollo Regional, Universidad Autónoma de Guerrero, Privada de Laurel 13, \\ Col. El Roble, Acapulco, Guerrero 39640, Mexico; fmira8906@gmail.com \\ * Correspondence: abel.cabrera@urv.cat
}

Received: 4 September 2020; Accepted: 16 October 2020; Published: 20 October 2020

\begin{abstract}
Let $G$ be a graph with no isolated vertex and $f: V(G) \rightarrow\{0,1,2\}$ a function. If $f$ satisfies that every vertex in the set $\{v \in V(G): f(v)=0\}$ is adjacent to at least one vertex in the set $\{v \in V(G): f(v)=2\}$, and if the subgraph induced by the set $\{v \in V(G): f(v) \geq 1\}$ has no isolated vertex, then we say that $f$ is a total Roman dominating function on $G$. The minimum weight $\omega(f)=\sum_{v \in V(G)} f(v)$ among all total Roman dominating functions $f$ on $G$ is the total Roman domination number of $G$. In this article we study this parameter for the rooted product graphs. Specifically, we obtain closed formulas and tight bounds for the total Roman domination number of rooted product graphs in terms of domination invariants of the factor graphs involved in this product.
\end{abstract}

Keywords: total Roman domination; total domination; rooted product graph

\section{Introduction}

The study of domination-related parameters in product graphs is one of the most important and attractive areas of domination theory in graphs. Among the principal attractions of this area, Vizing's Conjecture [1] is possibly the most popular open problem for the theory of domination in graphs. This conjecture asserts that the domination number of the Cartesian product of two graphs is at least equal to the product of their domination numbers. In recent years, several kinds of domination-related parameters in product graphs have been studied. For instance, we cite the following works: total domination [2] and total Roman domination [3] in direct product graphs; Roman domination in lexicographic product graphs [4]; domination-related parameters like classical domination, Roman domination, independence domination, connected domination, convex domination and super domination in rooted product graphs [5]; and Roman domination in Cartesian and strong product graphs [6]. In this article, we study a very well-known variant of domination (total Roman domination) for the case of rooted product graphs.

We consider $G=(V(G), E(G))$ as a simple graph. Given a vertex $v$ of a graph $G, N(v)$ will denote the open neighborhood of $v$ in $G$, i.e., the set of vertices of $G$ adjacent to $v$. The closed neighborhood, denoted by $N[v]$, equals $N(v) \cup\{v\}$. Given a set $S \subseteq V(G)$, its open neighborhood is the set $N(S)=\cup_{v \in S} N(v)$, and its closed neighborhood is the set $N[S]=N(S) \cup S$. As usual, the graph obtained from $G$ by removing all the vertices in $S \subseteq V(G)$ will be denoted by $G-S$. If $S=\{v\}$, for some vertex $v$, then we simple write $G-v$. 
The degree of a vertex $v$ in $G$ is $d(v)=|N(v)|$. A vertex $v \in V(G)$ is universal if $d(v)=|V(G)|-1$. A leaf of $G$ is a vertex $v$ with degree $d(v)=1$. A support of $G$ is a vertex adjacent to a leaf and a strong leaf is a leaf at distance two from another leaf. The sets of leaves, support vertices and strong leaves are denoted by $L(G), S(G)$ and $L_{S}(G)$, respectively.

A set $S \subseteq V(G)$ is a dominating set of $G$ if $N[S]=V(G)$. The domination number of $G$, denoted by $\gamma(G)$, is the minimum cardinality among all dominating sets of $G$. A dominating set with cardinality $\gamma(G)$ is called a $\gamma(G)$-set. A similar agreement will be assumed when referring to optimal functions (or sets) associated to another parameter. Moreover, a dominating set $S$ of $G$ is a total dominating set (TDS) of $G$ if $N(S)=V(G)$, i.e., every vertex of $G$ is adjacent to at least one vertex in $S$. The minimum cardinality among all TDSs of $G$ is the total domination number of $G$, and is denoted $\gamma_{t}(G)$. For more information about these two parameters, see the following books [7-9].

Let $G$ be a graph and $f: V(G) \rightarrow\{0,1,2\}$ a function. Observe that $f$ induces three sets $V_{0}, V_{1}$ and $V_{2}$ such that $V_{i}=\{v \in V(G): f(v)=i\}$ for $i=0,1,2$. Hence, we will write $f\left(V_{0}, V_{1}, V_{2}\right)$ instead $f: V(G) \rightarrow\{0,1,2\}$ so as to refer to $f$. If $D$ is a subset of vertices of $G$, then $f(D)=\sum_{v \in D} f(v)$. Hence, we define the weight of $f$ as $\omega(f)=f(V(G))=\left|V_{1}\right|+2\left|V_{2}\right|$.

One of the domination variants which has been intensively studied in the last two decades concerns the so-called Roman domination, which was formally presented in [10] and was motivated in part by the defensive strategy of the Roman Empire decreed by Constantine (see [11,12]). Several applications of Roman domination were shown in [13]. The Roman domination in graphs is a useful tool for modeling optimization problems such as facility location problems (building a new hospital, fire station, or restaurant), planning of defense strategies, surveillance related problems, communication networks, etc. Given a graph $G$, a function $f\left(V_{0}, V_{1}, V_{2}\right)$ is called a Roman dominating function (RDF) if every vertex in $V_{0}$ is adjacent to a vertex in $V_{2}$. The minimum weight among all RDFs on $G$ is the Roman domination number, and is denoted $\gamma_{R}(G)$. Several well-known results relate the Roman domination number with the (total) domination number. For instance, $\gamma_{t}(G) \leq \gamma_{R}(G)$ (see [14]) and $\gamma_{R}(G) \leq 2 \gamma(G)$ (see [10]). Further results on Roman domination can be found for example in [13-16].

The concept of total Roman domination in graphs was formally presented in [17], although it was previously introduced in [18] in a more general form. A total Roman dominating function (TRDF) on a graph $G$ without isolated vertices is an $\operatorname{RDF} f\left(V_{0}, V_{1}, V_{2}\right)$ such that $V_{1} \cup V_{2}$ is a total dominating set of $G$. The minimum weight among all TRDFs on $G$ is the total Roman domination number, and is denoted $\gamma_{t R}(G)$. As expected, (total) domination number, Roman domination number and total Roman domination number are related. The following relationships are proofs of this: $\gamma_{R}(G) \leq \gamma_{t R}(G) \leq 2 \gamma_{t}(G)$ (see [17]) and $\gamma_{t R}(G) \leq \gamma_{R}(G)+\gamma(G)$ (see [19]). Further results on total Roman domination can be found for example, in [19-22].

The complexity of the total Roman domination number was studied in [18]. The authors showed that the decision problem related to total Roman domination number is NP-hard even when restricted to bipartite graphs and chordal graphs. This suggests finding closed formulas or giving tight bounds on this domination invariant for special families of graphs. These studies are attractive, for instance, for the product graphs, and as previously shown, several studies in this regard have been carried out.

Our goal with this paper is to make some contributions to the study of total Roman domination number for the case of rooted product graphs. In that regard, in the next section we state the intervals in which this parameter can be found in a rooted product graph. Furthermore, we obtain closed formulas and tight bounds for this parameter.

\section{Main Results in Rooted Product Graphs}

Given a graph $G$ of order $n$ and a graph $H$ with $\operatorname{root} v \in V(H)$, the rooted product graph $G \circ_{v} H$ is defined as the graph obtained from $G$ and $H$ by taking one copy of $G$ and $n$ copies of $H$ and identifying the $i$ th-vertex of $G$ with vertex $v$ in the $i t h$-copy of $H$ for every $i \in\{1, \ldots, n\}$ [23]. Figure 1 shows an example of rooted product graph $P_{4} \circ_{v} H$, where $P_{4}$ is the path graph of order four. 


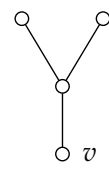

$H$

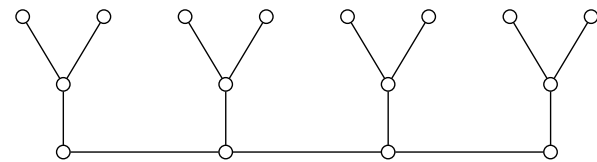

$\mathrm{P}_{4} \circ_{v} H$

Figure 1. The rooted product graph $P_{4} \circ_{v} H$.

For every $x \in V(G), H_{x}$ will denote the copy of $H$ in $G \circ_{v} H$ containing $x$. The restriction of any $\gamma_{t R}\left(G \circ_{v} H\right)$-function $f$ to $V\left(H_{x}\right)$ will be denoted by $f_{x}$ and the restriction to $V\left(H_{x}\right) \backslash\{x\}$ will be denoted by $f_{x}^{-}$.

Now, we state some tools, which will be very useful throughout the article.

Lemma 1. Let $H$ be a graph without isolated vertices and $v \in V(H) \backslash S(H)$. Then

$$
\gamma_{t R}(H-v) \geq \gamma_{t R}(H)-2 .
$$

Moreover, if $\gamma_{t R}(H-v)=\gamma_{t R}(H)-2$, then the following conditions hold.

(i) $\quad f(N(v))=0$ for every $\gamma_{t R}(H-v)$-function $f$.

(ii) There exists a $\gamma_{t R}(H)$-function $g_{0}$ satisfying $g_{0}(v)=0$.

(iii) There exists a $\gamma_{t R}(H)$-function $g_{1}$ satisfying $g_{1}(v)=1$.

Proof. Let $f$ be a $\gamma_{t R}(H-v)$-function and $w \in N(v)$. Let $h$ be a function on $H$ defined as follows: $h(w)=\min \{f(w)+1,2\}, h(v)=1$ and $h(u)=f(u)$ whenever $u \in V(H) \backslash\{v, w\}$. Observe that $h$ is a TRDF on $H$. Hence, $\gamma_{t R}(H-v)=\omega(f) \geq \omega(h)-2 \geq \gamma_{t R}(H)-2$, as desired.

From now on, we assume that $\gamma_{t R}(H-v)=\gamma_{t R}(H)-2$. Suppose that there exists $w \in N(v)$ such that $f(w)>0$. In such a case, we deduce that the function $h^{\prime}$, defined by $h^{\prime}(v)=0, h^{\prime}(w)=2$ and $h^{\prime}(u)=f(u)$ whenever $u \in V(H) \backslash\{v, w\}$, is a TRDF on $H$. This implies that $\gamma_{t R}(H) \leq$ $\omega\left(h^{\prime}\right) \leq \omega(f)+1=\gamma_{t R}(H)-1$, which is a contradiction. Thus, $f(N(v))=0$. Finally, if $w \in N(v)$, then the function $g_{0}$, defined by $g_{0}(v)=0, g_{0}(w)=2$ and $g_{0}(u)=f(u)$ whenever $u \in V(H) \backslash\{v, w\}$, is a $\gamma_{t R}(H)$-function. Analogously, the function $g_{1}$, defined by $g_{1}(v)=1, g_{1}(w)=1$ and $g_{1}(u)=f(u)$ whenever $u \in V(H) \backslash\{v, w\}$, is a $\gamma_{t R}(H)$-function as well, which completes the proof.

Following is a theorem bounding $\gamma_{t R}(H-v)$, for a particular vertex $v \in V(H)$. Before stating this, we shall need the next remark.

Remark 1. Let $H$ be a graph without isolated vertices. If $v \in L_{S}(H)$, then there exists a $\gamma_{t R}(H)$-function $f$ such that $f(v)=0$.

Proof. Let $f\left(V_{0}, V_{1}, V_{2}\right)$ be a $\gamma_{t R}(H)$-function such that $f(L(H))$ is minimum among all $\gamma_{t R}(H)$-functions. Let $N(v)=\{u\}$. Since $u \in S(H)$, we have that $u \in V_{1} \cup V_{2}$. If $u \in V_{1}$, then $N(u) \cap L(H) \subseteq V_{1}$. As $|N(u) \cap L(H)| \geq 2$, it follows that the function $f^{\prime}$, defined by $f^{\prime}(u)=2, f^{\prime}(v)=0$ and $f^{\prime}(x)=f(x)$ whenever $x \in V(H) \backslash\{u, v\}$ is a $\gamma_{t R}(H)$-function such that $f^{\prime}(L(H))<f(L(H))$, which is a contradiction. Hence, $u \in V_{2}$, which implies that $f(N(u) \cap L(H)) \leq 1$. So, and without loss of generality, we can assume that $f(v)=0$ as $|N(u) \cap L(H)| \geq 2$. Therefore, the proof is complete.

Theorem 1. Let $H$ be a graph without isolated vertices. If $v \in L_{s}(H)$, then

$$
\gamma_{t R}(H)-1 \leq \gamma_{t R}(H-v) \leq \gamma_{t R}(H) .
$$

Proof. By Remark 1 there exists a $\gamma_{t R}(H)$-function $f$ such that $f(v)=0$. Hence, $f$ restricted to $V(H) \backslash\{v\}$ is a TRDF on $H-v$. Thus, $\gamma_{t R}(H-v) \leq f(V(H) \backslash\{v\})=\omega(f)=\gamma_{t R}(H)$. Moreover, 
let $g$ be a $\gamma_{t R}(H-v)$-function and let $u$ be the support vertex associated to $v$ in $H$. Since $g(u)>0$, we have that the function $g^{\prime}$, defined by $g^{\prime}(v)=1$ and $g^{\prime}(x)=g(x)$ whenever $x \in V(H) \backslash\{v\}$, is a TRDF on $H$. So, $\gamma_{t R}(H) \leq \omega\left(g^{\prime}\right)=\omega(g)+1=\gamma_{t R}(H-v)+1$, which completes the proof.

Next, we expose a result for the Roman domination number of rooted product graphs given by Kuziak et al. in [5], which will be used later in the paper.

Theorem 2. [5] Let $G$ be a graph of order $n \geq 2$. Then for any graph $H$ with root $v$ and at least two vertices,

$$
n\left(\gamma_{R}(H)-1\right)+\gamma(G) \leq \gamma_{R}(G \circ v) \leq n \gamma_{R}(H) .
$$

We continue this section with a useful lemma.

Lemma 2. Let $f\left(V_{0}, V_{1}, V_{2}\right)$ be a $\gamma_{t R}\left(G \circ \circ_{v} H\right)$-function. The following two statements hold for any vertex $x \in V(G)$.

(i) $\quad \omega\left(f_{x}\right) \geq \gamma_{t R}(H)-2$.

(ii) If $\omega\left(f_{x}\right)=\gamma_{t R}(H)-2$, then $N[x] \cap V\left(H_{x}\right) \subseteq V_{0}$.

Proof. Let $x \in V(G)$. Observe that every vertex in $V_{0} \cap\left(V\left(H_{x}\right) \backslash\{x\}\right)$ has a neighbour in $V_{2} \cap V\left(H_{x}\right)$ and every vertex in $\left(V_{1} \cup V_{2}\right) \cap\left(V\left(H_{x}\right) \backslash\{x\}\right)$ has a neighbour in $\left(V_{1} \cup V_{2}\right) \cap V\left(H_{x}\right)$. First, we proceed to prove (i). Suppose that $\omega\left(f_{x}\right) \leq \gamma_{t R}(H)-3$ and let $w \in N(x) \cap V\left(H_{x}\right)$. Let $g$ be a function on $H_{x}$ defined as follows: $g(w)=2$ and $g(u)=f_{x}(u)$ whenever $u \in V\left(H_{x}\right) \backslash\{w\}$. It is easy to see that $g$ is a TRDF on $H_{x}$. Hence, $\gamma_{t R}\left(H_{x}\right) \leq \omega(g) \leq \omega\left(f_{x}\right)+2 \leq \gamma_{t R}(H)-1$, which is a contradiction as $H_{x} \cong H$. Thus, $\omega\left(f_{x}\right) \geq \gamma_{t R}(H)-2$, which completes the proof of (i).

Now, we proceed to prove (ii). Assume that $\omega\left(f_{x}\right)=\gamma_{t R}(H)-2$. Suppose that $x \in V_{1} \cup V_{2}$. Let $w \in N(x) \cap V\left(H_{x}\right)$ and we define the function $f^{\prime}$ as follows: $f^{\prime}(w)=\min \{f(w)+1,2\}$ and $f^{\prime}(u)=f(u)$ whenever $u \in V\left(H_{x}\right) \backslash\{w\}$. Notice that $f^{\prime}$ is a TRDF on $H_{x}$ such that $\omega\left(f^{\prime}\right) \leq \gamma_{t R}(H)-1$, which is a contradiction. Thus, $x \in V_{0}$. Finally, suppose there exists $w \in N(x) \cap V\left(H_{x}\right) \cap\left(V_{1} \cup V_{2}\right)$. In such a case, we define the function $f^{\prime \prime}$ as follows: $f^{\prime \prime}(w)=2$ and $f^{\prime \prime}(u)=f(u)$ whenever $u \in V\left(H_{x}\right) \backslash\{w\}$. As in the case above, $f^{\prime \prime}$ is a TRDF on $H_{x}$ such that $\omega\left(f^{\prime \prime}\right) \leq \gamma_{t R}(H)-1$, which is again a contradiction. Thus, $N(x) \cap V\left(H_{x}\right) \subseteq V_{0}$, and the proof is complete.

From Lemma 2-(i) we deduce that any $\gamma_{t R}\left(G \circ_{v} H\right)$-function $f$ generates three sets $\mathcal{A}_{f}, \mathcal{B}_{f}, \mathcal{C}_{f}$ of $V(G)$ as follows.

$$
\begin{aligned}
& \mathcal{A}_{f}=\left\{x \in V(G): \omega\left(f_{x}\right) \geq \gamma_{t R}(H)\right\}, \\
& \mathcal{B}_{f}=\left\{x \in V(G): \omega\left(f_{x}\right)=\gamma_{t R}(H)-1\right\}, \\
& \mathcal{C}_{f}=\left\{x \in V(G): \omega\left(f_{x}\right)=\gamma_{t R}(H)-2\right\} .
\end{aligned}
$$

Proposition 1. Let $f$ be a $\gamma_{t R}\left(G \circ_{v} H\right)$-function. If $\mathcal{C}_{f} \neq \varnothing$, then $v \in V(H) \backslash S(H)$ and

$$
\gamma_{t R}(H-v)=\gamma_{t R}(H)-2 .
$$

Proof. By Lemma 2-(ii), if $x \in \mathcal{C}_{f}$, then $f(x)=0$ and $f(y)=0$ for every $y \in N(x) \cap V\left(H_{x}\right)$. This implies that $x \in V\left(H_{x}\right) \backslash S\left(H_{x}\right)$, and also that $f$ restricted to $V\left(H_{x}\right) \backslash\{x\}$ is a TRDF on $H_{x}-x$ of weight $\gamma_{t R}(H)-2$. Since $H \cong H_{x}$, it follows that $v \in V(H) \backslash S(H)$ and $\gamma_{t R}(H-v)=$ $\gamma_{t R}\left(H_{x}-x\right) \leq \gamma_{t R}(H)-2$. Finally, by Lemma 1 we obtain that $\gamma_{t R}(H-v)=\gamma_{t R}(H)-2$, which completes the proof.

Theorem 3. Let $G$ and $H$ be two graphs without isolated vertices. If $|V(G)|=n$ and $v \in V(H)$, then the following statements hold. 
(i) $\gamma_{t R}\left(G \circ \circ_{v} H\right) \leq n \gamma_{t R}(H)$.

(ii) If $v \in V(H) \backslash S(H)$, then $\gamma_{t R}(G \circ v H) \leq \gamma_{t R}(G)+n \gamma_{t R}(H-v)$.

Proof. First, we proceed to prove (i). Notice that, from any $\gamma_{t R}(H)$-function, we can construct a TRDF on $G \circ_{v} H$ of weight $n \gamma_{t R}(H)$. Thus $\gamma_{t R}\left(G \circ_{v} H\right) \leq n \gamma_{t R}(H)$, which completes the proof of (i).

Finally, we proceed to prove (ii). Assume that $v \in V(H) \backslash S(H)$. Observe that, from any $\gamma_{t R}(G)$-function and any $\gamma_{t R}(H-v)$-function we can construct a TRDF on $G \circ_{v} H$ of weight at most $\gamma_{t R}(G)+n \gamma_{t R}(H-v)$, which completes the proof.

The next theorem, which we can consider as one of the main results of this paper, states the intervals in which the total Roman domination number of a rooted product graph can be found.

Theorem 4. Let $G$ and $H$ be two graphs without isolated vertices. If $|V(G)|=n$ and $v \in V(H)$, then one of the following conditions holds.

(i) $\gamma_{t R}\left(G \circ \circ_{v} H\right)=n \gamma_{t R}(H)$.

(ii) $n\left(\gamma_{t R}(H)-1\right) \leq \gamma_{t R}\left(G \circ \circ_{v} H\right) \leq \gamma_{t R}(G)+n\left(\gamma_{t R}(H)-1\right)$.

(iii) $\gamma_{t}(G)+n\left(\gamma_{t R}(H)-2\right) \leq \gamma_{t R}(G \circ v) \leq \gamma_{t R}(G)+n\left(\gamma_{t R}(H)-2\right)$.

Proof. Let $f\left(V_{0}, V_{1}, V_{2}\right)$ be a $\gamma_{t R}\left(G \circ_{v} H\right)$-function and we consider the sets $\mathcal{A}_{f}, \mathcal{B}_{f}$ and $\mathcal{C}_{f}$ defined above. Now, we analyze the following cases.

Case 1. $\mathcal{B}_{f} \cup \mathcal{C}_{f}=\varnothing$. By definition we deduce that $\omega\left(f_{x}\right) \geq \gamma_{t R}(H)$ for every $x \in V(G)$ and, as a consequence, $\gamma_{t R}\left(G \circ_{v} H\right) \geq n \gamma_{t R}(H)$. By Theorem 3, condition (i) follows.

Case 2. $\mathcal{B}_{f} \neq \varnothing$ and $\mathcal{C}_{f}=\varnothing$. By definition we deduce that $\omega\left(f_{x}\right) \geq \gamma_{t R}(H)-1$ for every $x \in V(G)$ and, as a consequence, $\gamma_{t R}\left(G \circ \circ_{v} H\right) \geq n\left(\gamma_{t R}(H)-1\right)$. We only need to prove that $\gamma_{t R}\left(G \circ \circ_{v} H\right) \leq$ $\gamma_{t R}(G)+n\left(\gamma_{t R}(H)-1\right)$. Let $h$ be a $\gamma_{t R}(G)$-function and $u \in \mathcal{B}_{f}$. From $f, u$ and $h$, we define a function $g$ on $G \circ_{v} H$ as follows. For every vertex $x \in V(G)$, the restriction of $g$ to $V\left(H_{x}\right) \backslash\{x\}$ is induced from $f_{u}^{-}$, and we set $g(x)=\min \{f(x)+h(x), 2\}$. Notice that $g$ is a TRDF on $G \circ_{v} H$ of weight $\omega(g) \leq \gamma_{t R}(G)+n\left(\gamma_{t R}(H)-1\right)$, concluding that $\gamma_{t R}(G \circ v) \leq \gamma_{t R}(G)+n\left(\gamma_{t R}(H)-1\right)$. Hence, condition (ii) follows.

Case 3. $\mathcal{B}_{f}=\varnothing$ and $\mathcal{C}_{f} \neq \varnothing$. By definition we obtain that

$$
\begin{aligned}
\gamma_{t R}\left(G \circ_{v} H\right) & =\sum_{x \in \mathcal{A}_{f}} \omega\left(f_{x}\right)+\sum_{x \in \mathcal{C}_{f}} \omega\left(f_{x}\right) \\
& \geq \sum_{x \in \mathcal{A}_{f}} \gamma_{t R}(H)+\sum_{x \in \mathcal{C}_{f}}\left(\gamma_{t R}(H)-2\right) \\
& =2\left|\mathcal{A}_{f}\right|+\sum_{x \in V(G)}\left(\gamma_{t R}(H)-2\right) \\
& =2\left|\mathcal{A}_{f}\right|+n\left(\gamma_{t R}(H)-2\right)
\end{aligned}
$$

From Lemma 2-(ii) we have that every vertex $x \in \mathcal{C}_{f}$ satisfies that $N[x] \cap V\left(H_{x}\right) \subseteq V_{0}$. As $\mathcal{B}_{f}=\varnothing$, there exists a vertex $y \in \mathcal{A}_{f} \cap N(x) \cap V_{2}$. This implies that $\mathcal{A}_{f} \cap V_{2}$ dominates $\mathcal{C}_{f}$, and as a consequence, $\mathcal{A}_{f}$ is a dominating set of $G$. Hence, $\left|A_{f}\right| \geq \gamma(G)$. Combining the inequalities above, we deduce that $\gamma_{t R}\left(G \circ \circ_{v} H\right) \geq 2\left|\mathcal{A}_{f}\right|+n\left(\gamma_{t R}(H)-2\right) \geq 2 \gamma(G)+n\left(\gamma_{t R}(H)-2\right) \geq \gamma_{t}(G)+n\left(\gamma_{t R}(H)-2\right)$.

On the other hand, by Proposition 1 we have that $v \in V(H) \backslash S(H)$ and that $\gamma_{t R}(H-v)=$ $\gamma_{t R}(H)-2$. Moreover, Theorem 3-(ii) leads to $\gamma_{t R}(G \circ v) \leq \gamma_{t R}(G)+n \gamma_{t R}(H-v)=\gamma_{t R}(G)+$ $n\left(\gamma_{t R}(H)-2\right)$. Thus, condition (iii) follows.

Case 4. $\mathcal{B}_{f} \neq \varnothing$ and $\mathcal{C}_{f} \neq \varnothing$. By Proposition 1 and Theorem 3-(ii) it follows that $\gamma_{t R}(G \circ v) \leq$ $\gamma_{t R}(G)+n\left(\gamma_{t R}(H)-2\right)$. 
Finally, let us define a set $S \subseteq V(G)$ as follows. If $x \in \mathcal{A}_{f}$ then we choose one vertex $u_{x} \in N(x) \cap V(G)$ and set $x, u_{x} \in S$. For the other vertices, if $x \in \mathcal{B}_{f}$ then we set $x \in S$. Now, we will prove that $S$ is a total dominating set of $G$. By definition of $S$, if $x \in \mathcal{A}_{f}$, then $x$ is adjacent to some vertex $y \in S$. Now, by Lemma 2-(ii), if $x \in C_{f}$ then there exists a vertex $y \in N(x) \cap\left(\mathcal{B}_{f} \cup \mathcal{A}_{f}\right)$, which implies that $y \in S$. If $x \in \mathcal{B}_{f}$, then it must have a neighbor $z \in V(G) \cap\left(V_{1} \cup V_{2}\right)$, otherwise $f_{x}$ is a TRDF on $H_{x}$ and so $\gamma_{t R}(H)=\gamma_{t R}\left(H_{x}\right) \leq \omega\left(f_{x}\right)=\gamma_{t R}(H)-1$, which is a contradiction. Hence, $z \in \mathcal{B}_{f} \cup \mathcal{A}_{f}$, which implies that $z \in S$, as required. Therefore, $S$ is a total dominating set of $G$ of cardinality at most $2\left|\mathcal{A}_{f}\right|+\left|\mathcal{B}_{f}\right|$ and, as a consequence,

$$
\begin{aligned}
\gamma_{t R}\left(G \circ \circ_{v} H\right) & =\sum_{x \in \mathcal{A}_{f}} \omega\left(f_{x}\right)+\sum_{x \in \mathcal{B}_{f}} \omega\left(f_{x}\right)+\sum_{x \in \mathcal{C}_{f}} \omega\left(f_{x}\right) \\
& \geq \sum_{x \in \mathcal{A}_{f}} \gamma_{t R}(H)+\sum_{x \in \mathcal{B}_{f}}\left(\gamma_{t R}(H)-1\right)+\sum_{x \in \mathcal{C}_{f}}\left(\gamma_{t R}(H)-2\right) \\
& =\left(2\left|\mathcal{A}_{f}\right|+\left|\mathcal{B}_{f}\right|\right)+\sum_{x \in V(G)}\left(\gamma_{t R}(H)-2\right) \\
& \geq|S|+n\left(\gamma_{t R}(H)-2\right) \\
& \geq \gamma_{t}(G)+n\left(\gamma_{t R}(H)-2\right) .
\end{aligned}
$$

So, condition (iii) follows, which completes the proof.

The bounds given in the theorem above are tight. To see this, we consider the following examples where $H_{1}$ is the graph shown in Figure 2 (we always assume that $G$ is a graph of order $n$ without isolated vertices). Recall that $P_{n}$ is the path graph of order $n$.

- If $v \in S\left(P_{4}\right)$, then $\gamma_{t R}\left(G \circ \rho_{v} P_{4}\right)=n \gamma_{t R}\left(P_{4}\right)=4 n$.

- If $v \in S\left(P_{3}\right)$, then $\gamma_{t R}\left(G \circ v P_{3}\right)=n\left(\gamma_{t R}\left(P_{3}\right)-1\right)=2 n$.

- If $v \in L\left(P_{3}\right)$ and $\gamma_{t R}(G)=\gamma_{R}(G)$, then $\gamma_{t R}\left(G \circ_{v} P_{3}\right)=\gamma_{t R}(G)+n\left(\gamma_{t R}\left(P_{3}\right)-1\right)=\gamma_{t R}(G)+2 n$.

- $\gamma_{t R}\left(G \circ H_{v} H_{1}\right)=\gamma_{t R}(G)+n\left(\gamma_{t R}\left(H_{1}\right)-2\right)=\gamma_{t R}(G)+4 n$.

- Theorem 5 gives some conditions to achieve the equality $\gamma_{t R}\left(G \circ \circ_{v} H\right)=\gamma_{t}(G)+n\left(\gamma_{t R}(H)-2\right)$. In this case we can take $H$, for example, as the graph given in Figure 3.

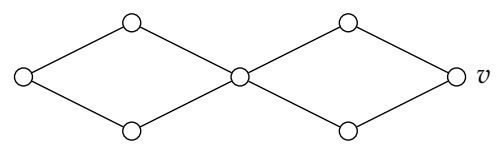

Figure 2. The graph $H_{1}$.

(a)

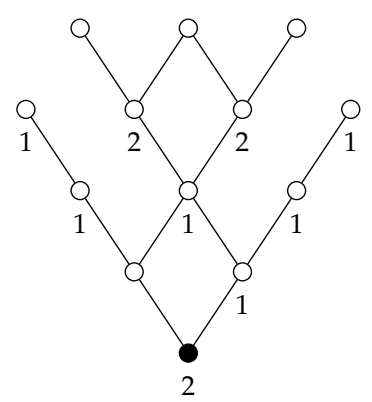

(b)

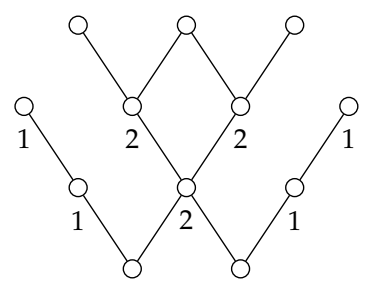

(c)

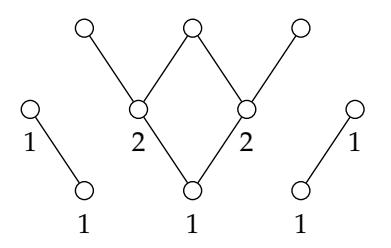

Figure 3. Graphs $H, H-v$ and $H-N[v]$ with different labelings (vertices with no drawn label have label zero) to show that $\gamma_{t R}(H)=12(\mathbf{a}), \gamma_{t R}(H-v)=10(\mathbf{b})$ and $\gamma_{t R}(H-N[v])=9$ (c).

Next, we analyse some particular cases. First, we consider the case in which $\gamma_{t R}(H-v)=\gamma_{t R}(H)-2$. 
Theorem 5. Let $G \nsubseteq \cup P_{2}$ be a graph of order $n$ without isolated vertices. If $H$ is a graph such that $\gamma_{t R}(H-v)=\gamma_{t R}(H)-2$ and $\gamma_{t R}(H-N[v])=\gamma_{t R}(H)-3$ for some $v \in V(H)$, then

$$
\gamma_{t R}\left(G \circ_{v} H\right)=\gamma_{t}(G)+n\left(\gamma_{t R}(H)-2\right)
$$

Proof. Let $g^{\prime}$ be a $\gamma_{t R}(H-v)$-function. By Lemma 1-(i), we may assume (without loss of generality) that $g^{\prime}(N(v))=0$. From $g^{\prime}$, we define the following function $g$ on $H$. If $x \in V(H-v)$ then $g(x)=g^{\prime}(x)$, and $g(v)=0$. Moreover, let $h^{\prime}$ be a $\gamma_{t R}(H-N[v])$-function and we consider the function $h$ on $H$ defined by $h(x)=h^{\prime}(x)$ if $x \in V(H-N[v]), h(x)=0$ if $x \in N(v)$, and $h(v)=2$.

Let $D$ be a $\gamma_{t}(G)$-set. From $D, g$ and $h$, we define the following function $f^{\prime}$ on $G \circ_{v} H$. For every $z \in D$, the restriction of $f^{\prime}$ to $V\left(H_{z}\right)$ is induced from $h$. Moreover, if $z \in V(G) \backslash D$, then the restriction of $f^{\prime}$ to $V\left(H_{z}\right)$ is induced from $g$. By the construction of $g$ and $h$, it is straightforward to see that $f^{\prime}$ is a TRDF on $G \circ_{v} H$. Thus,

$$
\begin{aligned}
\gamma_{t R}\left(G \circ_{v} H\right) & \leq \sum_{x \in D} \omega\left(f_{x}^{\prime}\right)+\sum_{x \in V(G) \backslash D} \omega\left(f_{x}^{\prime}\right) \\
& =\sum_{x \in D} \omega(h)+\sum_{x \in V(G) \backslash D} \omega(g) \\
& =\sum_{x \in D}\left(\gamma_{t R}(H)-1\right)+\sum_{x \in V(G) \backslash D}\left(\gamma_{t R}(H)-2\right) \\
& =|D|+\sum_{x \in V(G)}\left(\gamma_{t R}(H)-2\right) \\
& =\gamma_{t}(G)+n\left(\gamma_{t R}(H)-2\right) .
\end{aligned}
$$

We only need to prove that $\gamma_{t R}\left(G \circ_{v} H\right) \geq \gamma_{t}(G)+n\left(\gamma_{t R}(H)-2\right)$. Suppose that $\mathcal{C}_{f}=\varnothing$ by some $\gamma_{t R}\left(G \circ \circ_{v} H\right)$-function $f$. This implies that $n\left(\gamma_{t R}(H)-1\right) \leq \gamma_{t R}(G \circ v) \leq \gamma_{t}(G)+n\left(\gamma_{t R}(H)-2\right)$, which is a contradiction because $\gamma_{t}(G)<n$ (it is easy to see that $\gamma_{t}(G)=n$ if and only if $G \cong \cup P_{2}$ ). Therefore, $\mathcal{C}_{f} \neq \varnothing$ for any $\gamma_{t R}\left(G \circ_{v} H\right)$-function $f$. Hence, and by analogy to Cases 3 and 4 in the proof of Theorem 4, we deduce that $\gamma_{t R}(G \circ v) \geq \gamma_{t}(G)+n\left(\gamma_{t R}(H)-2\right)$, which completes the proof.

Notice that the premises given for the graph $H$ in the previous theorem lead to the existence of a $\gamma_{t R}(H)$-function $f$ satisfying $f(v)=2$. Since Lemma 1 does not guarantee the existence of a graph that satisfies such conditions, in Figure 3 we show a graph that does satisfy them.

Theorem 6. Let $G$ be a graph of order $n$ without isolated vertices and $H$ a graph such that $\gamma_{t R}(H-v)=\gamma_{t R}(H)-2$ for some $v \in V(H)$. If $g(v) \leq 1$ for every $\gamma_{t R}(H)$-function $g$, then

$$
\gamma_{t R}\left(G \circ_{v} H\right)=\gamma_{t R}(G)+n\left(\gamma_{t R}(H)-2\right) \text {. }
$$

Proof. By Theorem 3-(ii) and the equality $\gamma_{t R}(H-v)=\gamma_{t R}(H)-2$ we have that $\gamma_{t R}\left(G \circ_{v} H\right) \leq \gamma_{t R}(G)+n \gamma_{t R}(H-v)=\gamma_{t R}(G)+n\left(\gamma_{t R}(H)-2\right)$. To conclude the proof, we only need to prove that $\gamma_{t R}\left(G \circ_{v} H\right) \geq \gamma_{t R}(G)+n\left(\gamma_{t R}(H)-2\right)$. Let $f\left(V_{0}, V_{1}, V_{2}\right)$ be a $\gamma_{t R}\left(G \circ_{v} H\right)$-function. Let $\mathcal{A}_{f}=\mathcal{A}_{f} \cup \mathcal{A}_{f}^{>}$, where $\mathcal{A}_{f}^{\overline{\bar{f}}}=\left\{x \in \mathcal{A}_{f}: \omega(f)=\gamma_{t R}(H)\right\}$ and $\mathcal{A}_{f}^{>}=\mathcal{A}_{f} \backslash \mathcal{A}_{f}^{\overline{\bar{y}}}$. Since $g(v) \leq 1$ for every $\gamma_{t R}(H)$-function $g$, we deduce that $\mathcal{B}_{f} \subseteq V_{0} \cup V_{1}$ (otherwise, if there exists a vertex $x \in \mathcal{B}_{f} \cap V_{2}$, then we can obtain a $\gamma_{t R}\left(H_{x}\right)$-function $g$ with $g(x)=2$ by giving label 1 to some neighbor of $x$ in $H_{x}$, which is a contradiction as $\left.H_{x} \cong H\right)$. Moreover, if $x \in \mathcal{A}_{f}^{=} \cap V_{2}$, then $N(x) \cap V(G) \cap\left(V_{1} \cup V_{2}\right) \neq \varnothing$ (otherwise, $f_{x}$ would be a $\gamma_{t R}\left(H_{x}\right)$-function with $f_{x}(x)=2$, which contradicts the fact that $g(v) \leq 1$ for every $\gamma_{t R}(H)$-function $g$ ). Now, we define a function $h\left(V_{0}^{\prime}, V_{1}^{\prime}, V_{2}^{\prime}\right)$ on $G$.

(i) If $x \in \mathcal{A}_{f}^{>} \cup\left(\mathcal{A}_{f}^{\overline{\bar{f}}} \cap V_{2}\right)$, then we set $h(x)=2$.

(ii) If $x \in \mathcal{A}_{f}^{=} \backslash V_{2}$, then we set $h(x)=1$. 
(iii) If $x \in \mathcal{A}_{f}^{>} \cup\left(\mathcal{A}_{f}^{\overline{\bar{f}}} \backslash V_{2}\right)$, then we choose one vertex not previously labeled $u \in N(x) \cap V(G)$ (if it exists) and set $h(u)=1$.

(iv) We set $h(x)=f(x)$ for any other vertex $x \in V(G)$ not previously labeled.

We claim that $h\left(V_{0}^{\prime}, V_{1}^{\prime}, V_{2}^{\prime}\right)$ is a TRDF on $G$. First, we observe that if $x \in V_{0}^{\prime}$, then $x \in \mathcal{C}_{f} \cup\left(\mathcal{B}_{f} \cap V_{0}\right)$. Hence, there exists a vertex $y \in N(x) \cap V_{2} \cap \mathcal{A}_{f}$. Since $V_{2} \cap \mathcal{A}_{f} \subseteq V_{2}^{\prime}$, it follows that $y \in N(x) \cap V_{2}^{\prime}$, as desired.

We only need to prove that $V_{1}^{\prime} \cup V_{2}^{\prime}$ is a total dominating set of $G$. By definition of $h$, it is clear to see that every vertex in $V_{2}^{\prime} \cup\left(V_{1}^{\prime} \cap \mathcal{A}_{f}\right)$ is adjacent to some vertex in $V_{1}^{\prime} \cup V_{2}^{\prime}$. Now, let $x \in V_{1}^{\prime} \cap \mathcal{B}_{f}$. If $f\left(N(x) \cap V\left(H_{x}\right)\right)>0$, then $f_{x}$ is a TRDF on $H_{x}$ of weight $\gamma_{t R}(H)-1$, which is a contradiction. So, $f\left(N(x) \cap V\left(H_{x}\right)\right)=0$ and as $f$ is a TRDF on $G \circ_{v} H$, there exists a vertex $y \in N(x) \cap\left(\mathcal{A}_{f} \cup \mathcal{B}_{f}\right)$ such that $f(y)>0$. Hence, $y \in V_{1}^{\prime} \cup V_{2}^{\prime}$, as desired.

Thus, we conclude that $V_{1}^{\prime} \cup V_{2}^{\prime}$ is a total dominating set of $G$. So, $h$ is a TRDF on $G$, and as a consequence, $\gamma_{t R}(G) \leq \omega(h)=2\left|V_{2}^{\prime}\right|+\left|V_{1}^{\prime}\right| \leq 3\left|\mathcal{A}_{f}^{>}\right|+2\left|\mathcal{A}_{f}\right|+\left|\mathcal{B}_{f}\right|$. Therefore,

$$
\begin{aligned}
\gamma_{t R}\left(G \circ_{v} H\right) & =\sum_{x \in \mathcal{A}_{f}^{>}} \omega\left(f_{x}\right)+\sum_{x \in \mathcal{A}_{f}^{\overline{\bar{f}}}} \omega\left(f_{x}\right)+\sum_{x \in \mathcal{B}_{f}} \omega\left(f_{x}\right)+\sum_{x \in \mathcal{C}_{f}} \omega\left(f_{x}\right) \\
& \geq \sum_{x \in \mathcal{A}_{f}^{>}}\left(\gamma_{t R}(H)+1\right)+\sum_{x \in \mathcal{A}_{f}^{\bar{y}}} \gamma_{t R}(H)+\sum_{x \in \mathcal{B}_{f}}\left(\gamma_{t R}(H)-1\right)+\sum_{x \in \mathcal{C}_{f}}\left(\gamma_{t R}(H)-2\right) \\
& =\left(3\left|\mathcal{A}_{f}^{>}\right|+2\left|\mathcal{A}_{f}^{\overline{\bar{f}}}\right|+\left|\mathcal{B}_{f}\right|\right)+\sum_{x \in V(G)}\left(\gamma_{t R}(H)-2\right) \\
& \geq \gamma_{t R}(G)+n\left(\gamma_{t R}(H)-2\right) .
\end{aligned}
$$

Therefore, the proof is complete.

Now, we consider the cases in which either $v$ is a support vertex of $H$ or $\gamma_{t R}(H-v) \geq \gamma_{t R}(H)$. Before doing this, we shall need the following useful lemma.

Lemma 3. Let $f$ be a $\gamma_{t R}(G \circ v H)$-function. If there exists a vertex $x \in V(G)$ such that $f(x)>0$, then $\gamma_{t R}\left(G \circ \circ_{v} H\right) \leq n \omega\left(f_{x}\right)$.

Proof. Let $h$ be a function on $G \circ_{v} H$ defined from $f$ as follows. For every $z \in V(G)$, the restriction of $h$ to $V\left(H_{z}\right)$ is induced from $f_{x}$. Notice that $h$ is a TRDF on $G \circ_{v} H$, which implies that $\gamma_{t R}\left(G \circ \circ_{v} H\right) \leq n \omega\left(f_{x}\right)$, as desired.

Theorem 7. Let $G$ and $H$ be two graphs without isolated vertices and $|V(G)|=n$. If $v \in S(H)$ or $\gamma_{t R}(H-v) \geq \gamma_{t R}(H)$, then

$$
\gamma_{t R}\left(G \circ \circ_{v} H\right) \in\left\{n\left(\gamma_{t R}(H)-1\right), n \gamma_{t R}(H)\right\}
$$

Furthermore, if $\gamma_{t R}(H)=\gamma_{R}(H)$, then $\gamma_{t R}(G \circ v)=n \gamma_{t R}(H)$.

Proof. Let $f\left(V_{0}, V_{1}, V_{2}\right)$ be a $\gamma_{t R}(G \circ v H)$-function. By Theorem 3-(i) we have that $\omega(f) \leq n \gamma_{t R}(H)$. If $\omega(f)=n \gamma_{t R}(H)$, then we are done. In such a sense, we suppose that $\omega(f)<n \gamma_{t R}(H)$. This implies that there exists a vertex $x \in V(G)$ such that $\omega\left(f_{x}\right)<\gamma_{t R}(H)$, i.e., $x \in \mathcal{B}_{f} \cup \mathcal{C}_{f}$. If $f(x)=0$, then $x \notin S\left(H_{x}\right)$ and also $f_{x}^{-}$is a TRDF on $H_{x}-x$, which implies that $\gamma_{t R}(H-v)=\gamma_{t R}\left(H_{x}-x\right) \leq$ $\omega\left(f_{x}^{-}\right)=\omega\left(f_{x}\right)<\gamma_{t R}(H)$, which is a contradiction. Hence, $f(x)>0$. So, by Lemma 2-(ii) we deduce that $x \notin \mathcal{C}_{f}$, which implies that $x \in \mathcal{B}_{f}$. Moreover, since $f(x)>0$, by Lemma 3 we have that $\gamma_{t R}(G \circ v) \leq n \omega\left(f_{x}\right)=n\left(\gamma_{t R}(H)-1\right)$.

Now, we proceed to prove that $\gamma_{t R}\left(G \circ \circ_{v} H\right) \geq n\left(\gamma_{t R}(H)-1\right)$. If $v \in S(H)$, then $V(G) \subseteq S(G \circ H)$. This implies that $f(z)>0$ for every vertex $z \in V(G)$. So, by Lemma 2-(ii) we 
have that $\mathcal{C}_{f}=\varnothing$, which implies that $\gamma_{t R}(G \circ v)=\sum_{x \in \mathcal{A}_{f}} \omega\left(f_{x}\right)+\sum_{x \in \mathcal{B}_{f}} \omega\left(f_{x}\right) \geq n\left(\gamma_{t R}(H)-1\right)$. Moreover, if $v \in V(H) \backslash S(H)$, then by hypothesis we have that $\gamma_{t R}(H-v) \geq \gamma_{t R}(H)$. So, $\mathcal{C}_{f}=\varnothing$ by contrapositive of Proposition 1. Hence, as above, we deduce that $\gamma_{t R}\left(G \circ_{v} H\right)=$ $\sum_{x \in \mathcal{A}_{f}} \omega\left(f_{x}\right)+\sum_{x \in \mathcal{B}_{f}} \omega\left(f_{x}\right) \geq n\left(\gamma_{t R}(H)-1\right)$. Therefore, in both cases, the lower bound obtained leads to the equality $\gamma_{t R}(G \circ v)=n\left(\gamma_{t R}(H)-1\right)$, as desired.

Furthermore, if $\gamma_{t R}(H)=\gamma_{R}(H)$, then by Theorem 2 we have that $n\left(\gamma_{t R}(H)-1\right)+\gamma(G)=$ $n\left(\gamma_{R}(H)-1\right)+\gamma(G) \leq \gamma_{R}(G \circ v) \leq \gamma_{t R}(G \circ v)$. Since $\gamma(G) \geq 1$, the result above leads to $\gamma_{t R}\left(G \circ \circ_{v} H\right)=n \gamma_{t R}(H)$.

Observe that if $H$ is a nontrivial star graph or a double star graph (in both cases we consider the root $v \in S(H)$ ), then for any graph $G$ of order $n$ without isolated vertices we have that $\gamma_{t R}(G \circ v)=n\left(\gamma_{t R}(H)-1\right)$ and $\gamma_{t R}(G \circ v)=n \gamma_{t R}(H)$, respectively. These two particular cases belong to families of graphs that are analyzed in Theorems 9 and 12.

We continue with a result in which $v \in S(H)$ or $\gamma_{t R}(H-v) \geq \gamma_{t R}(H)-1$.

Theorem 8. Let $G$ and $H$ be two graphs without isolated vertices and $|V(G)|=n$. If $(v \in S(H)$ or $\left.\gamma_{t R}(H-v) \geq \gamma_{t R}(H)-1\right)$ and $\gamma_{t R}(H-N[v])=\gamma_{t R}(H)-3$, then

$$
\gamma_{t R}(G \circ v)=n\left(\gamma_{t R}(H)-1\right) .
$$

Proof. Let $h^{\prime}$ be a $\gamma_{t R}(H-N[v])$-function and let us consider the function $h$ on $H$ defined as follows. If $x \in V(H-N[v])$ then $h(x)=h^{\prime}(x)$, if $x \in N(v)$ then $h(x)=0$, and $h(v)=2$. Next, we define a function $f$ on $G \circ_{v} H$ from $h$. For every $z \in V(G)$, the restriction of $f$ to $V\left(H_{z}\right)$ is induced from $h$. By the construction of $h$, it is straightforward to see that $f$ is a TRDF on $G \circ_{v} H$. Thus,

$$
\begin{aligned}
\gamma_{t R}\left(G \circ \circ_{v} H\right) & \leq \sum_{x \in V(G)} \omega\left(f_{x}\right) \\
& =\sum_{x \in V(G)} \omega(h) \\
& =\sum_{x \in V(G)}\left(\omega\left(h^{\prime}\right)+2\right)=\sum_{x \in V(G)}\left(\gamma_{t R}(H)-1\right)=n\left(\gamma_{t R}(H)-1\right) .
\end{aligned}
$$

Therefore, Theorem 7 if $v \in S(H)$ or Proposition 1 and Lemma 2 if $\gamma_{t R}(H-v) \geq \gamma_{t R}(H)-1$, complete the proof.

In Figure 4 we show a graph $H$ which satisfies the premises of Theorem 8. In this case, $v \in S(H)$ and for any graph $G$ of order $n$ with no isolated vertex, we obtain that $\gamma_{t R}(G \circ v)=n\left(\gamma_{t R}(H)-1\right)=7 n$.

(a)

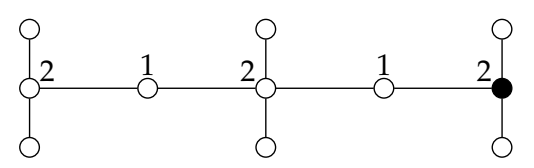

(b)

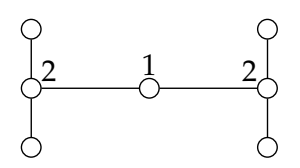

Figure 4. Graphs $H$ and $H-N[v]$ with different labellings (vertices with no drawn label have label zero) to show that $\gamma_{t R}(H)=8(\mathbf{a})$ and $\gamma_{t R}(H-N[v])=5(\mathbf{b})$.

Next, we proceed to discuss a particular case when $v \in S(H)$.

Theorem 9. Let $G$ and $H$ be two graphs without isolated vertices with $|V(G)|=n$ and $v \in S(H)$. If $N(v) \cap S(H) \neq \varnothing$ or $g(v)=1$ for every $\gamma_{t R}(H)$-function $g$, then

$$
\gamma_{t R}(G \circ v)=n \gamma_{t R}(H)
$$


Proof. Theorem 7 leads to $\gamma_{t R}\left(G \circ_{v} H\right) \in\left\{n\left(\gamma_{t R}(H)-1\right), n \gamma_{t R}(H)\right\}$. Hence, we only need to prove that $\gamma_{t R}(G \circ v H) \geq n \gamma_{t R}(H)$. Let $f\left(V_{0}, V_{1}, V_{2}\right)$ be a $\gamma_{t R}\left(G \circ \circ_{v} H\right)$-function such that $\left|V_{2}\right|$ is maximum among all $\gamma_{t R}(G \circ v)$-functions. Now, we analyse the following two cases.

Case 1. $N(v) \cap S(H) \neq \varnothing$. Let $y \in N(x) \cap S\left(H_{x}\right)$ for any $x \in V(G)$. As $x$ and $y$ are adjacent support vertices, it follows that $f(x)=f(y)=2$. Hence, $f_{x}$ is a TRDF on $H_{x}$, and as a consequence, $\omega\left(f_{x}\right) \geq \gamma_{t R}(H)$. Thus, $\gamma_{t R}(G \circ v H) \geq n \gamma_{t R}(H)$, as desired.

Case 2. $g(v)=1$ for every $\gamma_{t R}(H)$-function $g$. Since $v \in S(H)$, we have that $f(x) \geq 1$ for every vertex $x \in V(G)$. So, Lemma 2 leads to $\mathcal{C}_{f}=\varnothing$. If there exists $y \in V(G)$ such that $f(y)=1$, then $f\left(h_{y}\right)=1$ for every $h_{y} \in N(y) \cap L\left(H_{y}\right)$. Thus, the function $f^{\prime}\left(V_{0}^{\prime}, V_{1}^{\prime}, V_{2}^{\prime}\right)$, defined by $f^{\prime}(y)=2$, $f^{\prime}\left(N(y) \cap L\left(H_{y}\right)\right)=0$ and $f^{\prime}(u)=f(u)$ otherwise, is a $\gamma_{t R}(G \circ v)$-function and satisfies that $\left|V_{2}^{\prime}\right|>\left|V_{2}\right|$, which is a contradiction. Therefore, $f(x)=2$ for every $x \in V(G)$. Moreover, if $\mathcal{B}_{f} \neq \varnothing$, then there exists a $\gamma_{t R}(H)$-function $g$ such that $g(v)=2$, which is a contradiction. Hence, $\mathcal{B}_{f}=\varnothing$, and as a consequence, $\gamma_{t R}\left(G \circ_{v} H\right) \geq n \gamma_{t R}(H)$, as desired.

Now, we analyse other cases for the vertex $v \in V(H)$.

Theorem 10. Let $G$ be a graph without isolated vertices of order $n$. Let $H$ be a graph such that $\gamma_{t R}(H-v)=\gamma_{t R}(H)$ for some $v \in V(H)$. If $g(v)=0$ for every $\gamma_{t R}(H)$-function $g$, then

$$
\gamma_{t R}(G \circ v)=n \gamma_{t R}(H)
$$

Proof. Let $f\left(V_{0}, V_{1}, V_{2}\right)$ be a $\gamma_{t R}\left(G \circ_{v} H\right)$-function. With the assumptions given, Proposition 1 leads to $\mathcal{C}_{f}=\varnothing$. Now, we analyse two cases. First, if $\mathcal{B}_{f}=\varnothing$, then by analogy to Case 1 in the proof of Theorem 4 we have that $\gamma_{t R}\left(G \circ_{v} H\right)=n \gamma_{t R}(H)$.

Second, we suppose that $\mathcal{B}_{f} \neq \varnothing$ and let $x \in \mathcal{B}_{f}$. If $f(x)=0$, then $f_{x}^{-}$is a TRDF on $H_{x}-x$, which implies that $\gamma_{t R}(H-v)=\gamma_{t R}\left(H_{x}-x\right) \leq \omega\left(f_{x}^{-}\right)=\omega\left(f_{x}\right)=\gamma_{t R}(H)-1$, which is a contradiction. Hence, $f(x)>0$. If there exists a vertex $u \in N(x) \cap V\left(H_{x}\right)$ such that $f(u)>0$, then $f_{x}$ is a TRDF on $H_{x}$ of weight $\omega\left(f_{x}\right)=\gamma_{t R}(H)-1$, which is a contradiction. Hence, $N(x) \cap V\left(H_{x}\right) \subseteq V_{0}$. Note that the function $g$, defined by $g(u)=1$ for some vertex $u \in N(x) \cap V\left(H_{x}\right)$ and $g(w)=f(w)$ for every $w \in V\left(H_{x}\right) \backslash\{u\}$, is a TRDF on $H_{x}$ of weight $\gamma_{t R}(H)$. So $g$ is a $\gamma_{t R}(H)$-function and satisfies that $g(v)>0$, which is again a contradiction. Hence, $\mathcal{B}_{f}=\varnothing$, and we are done.

Next, we consider the case in which the root $v$ is a strong leaf vertex of $H$.

Theorem 11. Let $G$ and $H$ be two graphs without isolated vertices and $|V(G)|=n$. If $v \in L_{S}(H)$, then

$$
\gamma_{t R}(G \circ v)=\left\{\begin{array}{cl}
n \gamma_{t R}(H) & \text { if } \gamma_{t R}(H-v)=\gamma_{t R}(H), \\
\gamma_{R}(G)+n\left(\gamma_{t R}(H)-1\right) & \text { otherwise. }
\end{array}\right.
$$

Proof. By Theorem 1 we have that $\gamma_{t R}(H-v) \in\left\{\gamma_{t R}(H), \gamma_{t R}(H)-1\right\}$. In such a sense, we consider the following two cases.

Case 1. $\gamma_{t R}(H-v)=\gamma_{t R}(H)$. Let $f\left(V_{0}, V_{1}, V_{2}\right)$ be a $\gamma_{t R}\left(G \circ_{v} H\right)$-function. By Proposition 1 we have that $\mathcal{C}_{f}=\varnothing$. Suppose that $\mathcal{B}_{f} \neq \varnothing$ and let $x \in \mathcal{B}_{f}$. If $f(x)=0$, then $f_{x}^{-}$is a TRDF on $H_{x}-x$, which implies that $\gamma_{t R}(H-v)=\gamma_{t R}\left(H_{x}-x\right) \leq \omega\left(f_{x}^{-}\right)=\omega\left(f_{x}\right)=\gamma_{t R}(H)-1$, which is a contradiction. Hence, $f(x)>0$. Let $\left\{u_{x}\right\}=N(x) \cap V\left(H_{x}\right)$. Since $v \in L_{s}(H)$, it follows that $u_{x} \in S\left(G \circ_{v} H\right)$. Hence $f\left(u_{x}\right)>0$, and so $f_{x}$ is a TRDF on $H_{x}$ of weight $\omega\left(f_{x}\right)=\gamma_{t R}(H)-1$, which is a contradiction. Therefore, $\mathcal{B}_{f}=\varnothing$, and so $\gamma_{t R}\left(G \circ_{v} H\right)=n \gamma_{t R}(H)$.

Case 2. $\gamma_{t R}(H-v)=\gamma_{t R}(H)-1$. First, we prove that $\gamma_{t R}(G \circ v) \leq \gamma_{R}(G)+n\left(\gamma_{t R}(H)-1\right)$. Let $u$ be the support vertex associated to $v$, i.e., $N(v)=\{u\}$, and let $g$ be any $\gamma_{t R}(H-v)$-function. Note that $g(u)>0$ as $u \in S(H-v)$. So, from $g$ and any $\gamma_{R}(G)$-function, we can define a TRDF 
on $G \circ_{v} H$ of weight at most $\gamma_{R}(G)+n \gamma_{t R}(H-v)$. Hence, $\gamma_{t R}(G \circ v) \leq \gamma_{R}(G)+n \gamma_{t R}(H-v)=$ $\gamma_{R}(G)+n\left(\gamma_{t R}(H)-1\right)$, as desired.

To conclude the proof, we only need to prove that $\gamma_{t R}\left(G \circ \circ_{v} H\right) \geq \gamma_{R}(G)+n\left(\gamma_{t R}(H)-1\right)$. Let $f\left(V_{0}, V_{1}, V_{2}\right)$ be a $\gamma_{t R}\left(G \circ \circ_{v} H\right)$-function. Since $N(V(G)) \backslash V(G) \subseteq S(G \circ v)$, we have that $f(y)>0$ for every vertex $y \in N(V(G)) \backslash V(G)$. Hence, Lemma 2-(ii) leads to $\mathcal{C}_{f}=\varnothing$. Now, we analyze two subcases.

Subcase 2.1. $\mathcal{B}_{f}=\varnothing$. In this subcase, by analogy to Case 1 in the proof of Theorem 4 , we have that $\gamma_{t R}\left(G \circ \circ_{v} H\right)=n \gamma_{t R}(H)$. Therefore, it follows that $n \gamma_{t R}(H)=\gamma_{t R}\left(G \circ \circ_{v} H\right) \leq \gamma_{R}(G)+n\left(\gamma_{t R}(H)-1\right)$, which implies that $\gamma_{R}(G)=n$, i.e., $G \cong \cup P_{2}$ (see [10]). Thus, $\gamma_{t R}(G \circ v)=\gamma_{R}(G)+n\left(\gamma_{t R}(H)-1\right)$, as desired.

Subcase 2.2. $\mathcal{B}_{f} \neq \varnothing$. Let $x \in \mathcal{B}_{f}$ and $\left\{u_{x}\right\}=N(x) \cap V\left(H_{x}\right)$. As $u_{x} \in S\left(G \circ_{v} H\right)$, it follows that $f\left(u_{x}\right)>0$. If $f(x)>0$, then $f_{x}$ is a TRDF on $H_{x}$ of weight $\gamma_{t R}(H)-1$, which is a contradiction. Hence, $f(x)=0$, and as a consequence, $f\left(u_{x}\right)=1$ (otherwise, if $f\left(u_{x}\right)=2$, then, as above, $f_{x}$ is a TRDF on $H_{x}$ of weight $\gamma_{t R}(H)-1$, which is a contradiction). Therefore, $\mathcal{B}_{f} \subseteq V_{0}$ and $\mathcal{A}_{f} \cap V_{2}$ dominates $\mathcal{B}_{f}$. This implies that $f$ restricted to $V(G)$ is an RDF on $G$, and so, $\gamma_{R}(G) \leq f(V(G))$. Now, we suppose that there exists a vertex $z \in \mathcal{A}_{f} \cap V_{2}$ such that $\omega\left(f_{z}\right)=\gamma_{t R}(H)$. Let $u_{z} \in N(z) \cap V\left(H_{z}\right)$. Since $u_{z} \in S(G \circ v)$, it follows that $f\left(u_{z}\right)>0$. This implies that the function $f_{z}$ is a $\gamma_{t R}\left(H_{z}\right)$-function because $H_{z} \cong H$. Now, we observe that the function $g$, defined by $g(z)=1$ and $g(x)=f_{z}(x)$ whenever $x \in V\left(H_{z}\right) \backslash\{z\}$, is a TRDF on $H_{z}$. Hence, $\gamma_{t R}\left(H_{z}\right) \leq \omega(g)=\omega\left(f_{z}\right)-1=\gamma_{t R}\left(H_{z}\right)-1$, which is a contradiction. Therefore, every vertex $z \in \mathcal{A}_{f} \cap V_{2}$ satisfies that $\omega\left(f_{z}\right)>\gamma_{t R}(H)$. Thus, as $\mathcal{C}_{f}=\varnothing$ and $\mathcal{B}_{f} \subseteq V_{0}$, we obtain that $\gamma_{t R}\left(G \circ_{v} H\right)=\sum_{x \in \mathcal{A}_{f}} \omega\left(f_{x}\right)+\sum_{x \in \mathcal{B}_{f}} \omega\left(f_{x}\right) \geq f(V(G))+n\left(\gamma_{t R}(H)-\right.$ $1) \geq \gamma_{R}(G)+n\left(\gamma_{t R}(H)-1\right)$, which completes the proof.

The following theorem states the total Roman domination number of the rooted product graph $G \circ_{v} H$, when the root $v$ is a universal vertex of $H$.

Theorem 12. Let $G$ be a graph without isolated vertices of order $n$. If $H$ is a graph with a universal vertex $v \in V(H)$, then

$$
\gamma_{t R}(G \circ v)=2 n .
$$

Proof. Let $f$ be a function defined as follows: $f(x)=2$ if $x \in V(G)$ and $f(x)=0$ whenever $x \in V\left(G \circ \circ_{v} H\right) \backslash V(G)$. It is clear to see that $f$ is a TRDF on $G \circ_{v} H$. Hence, $\gamma_{t R}\left(G \circ \circ_{v} H\right) \leq \omega(f)=2 n$. Since $\gamma_{t R}(H)=3$, we have that either $v \in S(H)$ or $\gamma_{t R}(H-v) \geq \gamma_{t R}(H)$. Thus, Theorem 7 leads to the desired result.

Any corona graph $G \odot G^{\prime}$ can be expressed as a rooted product graph $G \circ_{v} H$, where $H \cong K_{1}+G^{\prime}$ and $V\left(K_{1}\right)=\{v\}$. Therefore, the following result follows as an immediate consequence of the theorem above.

Theorem 13. Let $G$ be a graph of order $n$ without isolated vertices and $G^{\prime}$ any graph. Then

$$
\gamma_{t R}\left(G \odot G^{\prime}\right)=2 n .
$$

\section{Conclusions and Open Problems}

In this paper, we have studied the total Roman domination number of rooted product graphs $G \circ_{v} H$. In particular, we were able to give the intervals to which $\gamma_{t R}\left(G \circ_{v} H\right)$ belongs. In addition, we have obtained closed formulas for this parameter when certain conditions are imposed on the factor graphs $G$ and $H$ involved in this product.

Next, we propose some open problems, which we consider to be interesting.

- Characterize the graphs $G$ and $H$ (and the root $v$ ) that satisfy the following equalities. 


$$
\begin{array}{ll}
- & \gamma_{t R}\left(G \circ \circ_{v} H\right)=n \gamma_{t R}(H) . \\
- & \gamma_{t R}\left(G \circ \circ_{v} H\right)=\gamma_{t R}(G)+n\left(\gamma_{t R}(H)-1\right) . \\
- & \gamma_{t R}\left(G \circ \circ_{v} H\right)=n\left(\gamma_{t R}(H)-1\right) . \\
- & \gamma_{t R}\left(G \circ \circ_{v} H\right)=\gamma_{t R}(G)+n\left(\gamma_{t R}(H)-2\right) . \\
- & \gamma_{t R}\left(G \circ \circ_{v} H\right)=\gamma_{t}(G)+n\left(\gamma_{t R}(H)-2\right) .
\end{array}
$$

- $\quad$ Characterize the graphs $G$ and $H$ (and the root $v$ ) such that $\gamma_{t R}(G \circ v H)=\gamma_{R}(G \circ v H)$.

- Given a graph $G$ of order $n$ and a family of $n$ graphs $\mathcal{H}=\left\{H_{1}, \ldots, H_{n}\right\}$ with a set of root vertices $W=\left\{w_{1}, w_{2}, \ldots, w_{n}\right\}$, respectively, the generalized rooted product graph $G \circ_{W} \mathcal{H}$ is defined as the graph obtained from $G$ and $\mathcal{H}$, by identifying the vertex $v_{i}$ of $G$ with the root vertex $w_{i} \in W$ of $H_{i}$ for every $1 \leq i \leq n$. We propose to study the total Roman domination number of generalized rooted product graphs.

Author Contributions: All authors contributed equally to this work. Investigation, A.C.M., S.C.G., A.C.G. and F.A.H.M.; writing-review and editing, A.C.M., S.C.G., A.C.G. and F.A.H.M. All authors have read and agreed to the published version of the manuscript.

Funding: This research received no external funding.

Acknowledgments: We would like to thank the anonymous referees for their comments and suggestions towards improving our manuscript.

Conflicts of Interest: The authors declare no conflicts of interest.

\section{References}

1. Vizing, V.G. The Cartesian product of graphs. Vyčisl. Sist. 1963, 9, 30-43.

2. Dorbec, P.; Gravier, S.; Klavžar, S.; Špacapan, S. Some results on total domination in direct product graphs. Discuss. Math. Graph Theory 2006, 26, 103-112. [CrossRef]

3. Cabrera Martínez, A.; Kuziak, D.; Peterin, I.; Yero, I.G. Dominating the Direct Product of Two Graphs through Total Roman Strategies. Mathematics 2020, 8, 1438. [CrossRef]

4. S̆umenjak, T.K.; Pavlič, P.; Tepeh, A. On the Roman domination in the lexicographic product of graphs. Discret. Appl. Math. 2012, 160, 2030-2036. [CrossRef]

5. Kuziak, D.; Lemanska, M.; Yero, I.G. Domination-Related parameters in rooted product graphs. Bull. Malays. Math. Sci. Soc. 2019, 39, 199-217. [CrossRef]

6. Yero, I.G.; Rodríguez-Velázquez, J.A. Roman domination in cartesian product graphs and strong product graphs. Appl. Anal. Discr. Math. 2013, 7, 262-274.

7. Haynes, T.W.; Hedetniemi, S.T.; Slater, P.J. Domination in Graphs: Advanced Topics; Chapman and Hall/CRC Pure and Applied Mathematics Series; Marcel Dekker, Inc.: New York, NY, USA, 1998.

8. Haynes, T.W.; Hedetniemi, S.T.; Slater, P.J. Fundamentals of Domination in Graphs; Chapman and Hall/CRC Pure and Applied Mathematics Series; Marcel Dekker, Inc.: New York, NY, USA, 1998.

9. Henning, M.; Yeo, A. Total Domination in Graphs, Springer Monographs in Mathematics; Springer: New York, NY, USA, 2013.

10. Cockayne, E.J.; Dreyer, P.A., Jr.; Hedetniemi, S.M.; Hedetniemi, S.T. Roman domination in graphs. Discrete Math. 2004, 278, 11-22. [CrossRef]

11. Revelle, C.S.; Rosing, K.E. Defendens imperium romanum: A classical problem in military strategy. Am. Math. Mon. 2000, 107, 585-594. [CrossRef]

12. Stewart, I. Defend the Roman Empire! Sci. Am. 1999, 28, 136--139. [CrossRef]

13. Chambers, E.W.; Kinnersley, B.; Prince, N.; West, D.B. Extremal problems for Roman domination. SIAM J. Discrete Math. 2009, 23, 1575-1586. [CrossRef]

14. Chellali, M.; Haynes, T.W.; Hedetniemi, S.T. Roman and Total Domination. Quaest. Math. 2015, 38, 749-757. [CrossRef]

15. Favaron, O.; Karami, H.; Khoeilar, R.; Sheikholeslami, S.M. On the Roman domination number of a graph. Discret. Math. 2009, 309, 3447-3451. [CrossRef]

16. Rao, M.; Talon, A. The 2-domination and Roman domination numbers of grid graphs. Discret. Math. Theor. Comput. Sci. 2019, 21, 9.

17. Abdollahzadeh Ahangar, H.; Henning, M.A.; Samodivkin, V.; Yero, I.G. Total Roman domination in graphs. Appl. Anal. Discret. Math. 2016, 10, 501-517. [CrossRef] 
18. Liu, C.-H.; Chang, G.J. Roman domination on strongly chordal graphs. J. Comb. Optim. 2013, 26, 608-619. [CrossRef]

19. Cabrera Martínez, A.; Cabrera García, S.; Carrión García, A. Further results on the total Roman domination number of graphs. Mathematics 2020, 8, 349. [CrossRef]

20. Amjadi, J.; Sheikholeslami, S.M.; Soroudi, M. On the total Roman domination in trees. Discuss. Math. Graph Theory 2019, 39, 519-532. [CrossRef]

21. Cabrera Martínez, A.; Rodríguez-Velázquez, J.A. Closed formulas for the total Roman domination number of lexicographic product graphs. 2020, Manuscript.

22. Campanelli, N.; Kuziak, D. Total Roman domination in the lexicographic product of graphs. Discret. Appl. Math. 2019, 263, 88-95. [CrossRef]

23. Godsil, C.D.; McKay, B.D. A new graph product and its spectrum. Bull. Aust. Math. Soc. 1978, 18, $21-28$. [CrossRef]

Publisher's Note: MDPI stays neutral with regard to jurisdictional claims in published maps and institutional affiliations.

(C) 2020 by the authors. Licensee MDPI, Basel, Switzerland. This article is an open access article distributed under the terms and conditions of the Creative Commons Attribution (CC BY) license (http://creativecommons.org/licenses/by/4.0/). 